\title{
The influence of the cluster environment on the large-scale radio continuum emission of 8 Virgo cluster spirals
}

\author{
B. Vollmer ${ }^{1}$, M. Soida ${ }^{2}$, A. Chung ${ }^{3}$, R. Beck ${ }^{4}$, M. Urbanik ${ }^{2}$, K. T. Chyży ${ }^{2}$, \\ K. Otmianowska-Mazur ${ }^{2}$, and J. H. van Gorkom ${ }^{5}$
}

\author{
1 CDS, Observatoire astronomique de Strasbourg, UMR 7550, 11 rue de l'université, 67000 Strasbourg, France \\ e-mail: bvollmer@astro.u-strasbg.fr \\ 2 Astronomical Observatory, Jagiellonian University, Kraków, Poland \\ Smithsonian Astrophysical Observatory, 60 Garden Street, Cambridge, MA 02138, USA \\ ${ }^{4}$ Max-Planck-Institut für Radioastronomie, Auf dem Hügel 69, 53121 Bonn, Germany \\ 5 Department of Astronomy, Columbia University, 538 West 120th Street, New York, NY 10027, USA
}

Received 3 November 2009 / Accepted 11 January 2010

ABSTRACT

\begin{abstract}
The influence of the environment on the polarized and total power radio continuum emission of cluster spiral galaxies is investigated. We present deep scaled array VLA 20 and $6 \mathrm{~cm}$ observations including polarization of 8 Virgo spiral galaxies. These data are combined with existing optical, $\mathrm{HI}$, and $\mathrm{H} \alpha$ data. Ram pressure compression leads to sharp edges of the total power distribution at one side of the galactic disk. These edges coincide with HI edges. In edge-on galaxies the extraplanar radio emission can extend further than the HI emission. In the same galaxies asymmetric gradients in the degree of polarization give additional information on the ram pressure wind direction. The local total power emission is not sensitive to the effects of ram pressure. The radio continuum spectrum might flatten in the compressed region only for very strong ram pressure. This implies that neither the local star formation rate nor the turbulent small-scale magnetic field are significantly affected by ram pressure. Ram pressure compression occurs mainly on large scales ( $\gtrsim 1 \mathrm{kpc}$ ) and is primarily detectable in polarized radio continuum emission.
\end{abstract}

Key words. galaxies: interactions - galaxies: ISM - galaxies: magnetic fields - radio continuum: galaxies

\section{Introduction}

Radio continuum emission is due to relativistic electrons of density $N_{\mathrm{e}}$ gyrating around the interstellar magnetic field $B$ : $S \propto N_{\mathrm{e}} B_{\perp}^{2}$, where $S$ is the intensity of synchrotron emission and $B_{\perp}$ is the component of the total magnetic field in the sky plane. The galactic magnetic field can be divided into a small-scale and large-scale component compared to the resolution of the radio continuum observations, which is typically about $1 \mathrm{kpc}$ in nearby galaxies. The small-scale magnetic field is due to turbulent gas motions and is therefore tangled. The large-scale magnetic field is due to a galactic dynamo. Polarized emission is due to the regularly oriented, large-scale magnetic field, but it can also be caused by an alignment of anisotropic small-scale magnetic fields, produced by stretching and compression of smallscale magnetic structures. While the large-scale unidirectional fields yield a non-zero Faraday rotation, this is not the case for aligned anisotropic small-scale fields. The small-scale magnetic field is typically a factor of 2-5 larger than the regular largescale magnetic field in spiral arms and 1-2 times larger in the interarm regions (Beck 2001). Whenever there is enhanced turbulence due to enhanced star formation, the small-scale magnetic field is increased and the large-scale magnetic field is diminished (see, e.g., Beck 2007).

Deep VLA observations at $\lambda 6 \mathrm{~cm}$ have shown that the distribution of polarized radio continuum emission of Virgo cluster spiral galaxies is strongly asymmetric, with elongated ridges located in the outer galactic disk (Vollmer et al. 2004a,b; Chyży et al. 2006, 2007; Vollmer et al. 2007). These features are not found in similar observations of field galaxies, where the distribution of $\lambda 6 \mathrm{~cm}$ polarized emission is generally relatively symmetric and strongest in the interarm regions (Beck 2005). The polarized radio continuum emission is sensitive to compression and shear motions within the galactic disks occurring during the interaction between the galaxy and its cluster environment. These interactions can be of tidal nature (with the cluster potential: Byrd \& Valtonen 1990; Valluri 1993, rapid flybys of massive galaxies, galaxy "harassment": Moore et al. 1998) or hydrodynamic nature (ram pressure stripping: Gunn \& Gott 1972).

On the other hand, total radio continuum emission is sensitive to star formation which gives rise to the radio-FIR correlation (see, e.g. Murphy et al. 2008). Based on $1.4 \mathrm{GHz}$ radio continuum observations Gavazzi et al. (1991) showed that this correlation that is shared by spiral galaxies in a huge luminosity interval, is different for cluster and isolated galaxies: the cluster galaxies have an increased radio/FIR ratio. A similar increase has been observed at $4.85 \mathrm{GHz}$ and $10.55 \mathrm{GHz}$ (Niklas et al. 1995) for 6 out of 45 observed Virgo spirals including NGC 4388 and NGC 4438. Gavazzi \& Boselli (1999) studied the distribution of the radio/NIR luminosity (RLF) of late-type galaxies in 5 nearby galaxy clusters. They found that the RLF of Cancer, ACO 262 and Virgo are consistent with that of isolated galaxies. However, galaxies in ACO 1367 and Coma have their radio emissivity enhanced by a factor $\sim 5$ with respect to isolated objects. Multiple systems in the Coma cluster bridge also show an enhanced radio/NIR emission. Gavazzi \& Boselli (1999) argue that the latter effect is due to increased star formation caused by tidal interactions, whereas the enhanced radio/NIR ratio in 
Table 1. Integration times and rms.

\begin{tabular}{|c|c|c|c|c|c|c|c|c|c|c|}
\hline Galaxy name & $\begin{array}{l}m_{\mathrm{B}}{ }^{1} \\
(\mathrm{mag}) \\
\end{array}$ & $\begin{array}{c}i^{2} \\
(\operatorname{deg}) \\
\end{array}$ & $\begin{array}{l}\text { Dist. }^{3} \\
(\mathrm{deg}) \\
\end{array}$ & $\begin{array}{c}\text { Integration } \\
\text { time }(6 \mathrm{~cm}) \\
(\mathrm{h}: \mathrm{min}) \\
\end{array}$ & $\begin{array}{c}\text { Integration } \\
\text { time }(20 \mathrm{~cm}) \\
(\mathrm{h}: \mathrm{min})\end{array}$ & $\begin{array}{c}\text { Bandwidth } \\
(20 \mathrm{~cm}) \\
(\mathrm{MHz}) \\
\end{array}$ & $\begin{array}{c}\text { Rms TP6 cm } \\
(\mu \mathrm{Jy} / \\
\text { beam })\end{array}$ & $\begin{array}{c}\text { Rms TP20 cm } \\
(\mu \mathrm{Jy} / \\
\text { beam }) \\
\end{array}$ & $\begin{array}{c}\text { Rms PI6 cm } \\
(\mu \mathrm{Jy} / \\
\text { beam })\end{array}$ & $\begin{array}{c}\text { Rms PI20 cm } \\
(\mu \mathrm{Jy} / \\
\text { beam })\end{array}$ \\
\hline NGC 4321 & 10.02 & $27^{a}$ & 4.0 & $7: 45$ & $0: 30$ & $2 \times 50$ & 10 & 110 & 9 & 35 \\
\hline NGC 4388 & 11.87 & $77^{a}$ & 1.3 & $9: 25$ & 8:00 & VIVA & 60 & 300 & 9 & 200 \\
\hline NGC 4396 & 13.07 & $72^{b}$ & 3.5 & 8:00 & $0: 30$ & $2 \times 50$ & 8 & 60 & 9 & 25 \\
\hline NGC 4402 & 12.64 & $74^{c}$ & 1.4 & $5: 00$ & $8: 00$ & VIVA & 21 & 80 & 13 & 100 \\
\hline NGC 4438 & 11.12 & $68^{a} / 85^{c}$ & 1.0 & $7: 45$ & 8:00 & VIVA & 30 & 60 & 14 & 300 \\
\hline NGC 4501 & 10.50 & $57^{a}$ & 2.0 & $3: 55$ & $5: 00$ & VIVA & 14 & 220 & 11 & 50 \\
\hline NGC 4535 & 10.73 & $43^{a}$ & 4.3 & 9:00 & $0: 30$ & $2 \times 50$ & 9 & 80 & 9 & 35 \\
\hline NGC 4654 & 11.31 & $51^{a}$ & 3.4 & $7: 50$ & $0: 30$ & $2 \times 50$ & 9 & 50 & 9 & 30 \\
\hline
\end{tabular}

Notes. ${ }^{(1)}$ This research has made use of the GOLD Mine Database (Gavazzi et al. 2003); (2) inclination angle; ${ }^{(3)}$ distance from M 87; ${ }^{(a)}$ from Cayatte et al. (1990); ${ }^{(b)}$ from NED; ${ }^{(c)}$ from Kenney et al. (1995).

ACO 1367 and Coma is due to ram pressure compression of the magnetic field. Murphy et al. (2009) investigated the radio-FIR relation of Virgo cluster galaxies. They showed that ram pressure affected galaxies have global radio flux densities that are enhanced by a factor of 2-3 compared to isolated galaxies.

The typical spectral slope of synchrotron emission from spiral galaxies between $1.4 \mathrm{GHz}$ and $4.85 \mathrm{GHz}$ is $S \propto v^{-0.8}$ (Gioia et al. 1982; Klein 1990). Völk \& Xu (1994) proposed that a shock-induced reacceleration of relativistic electrons during ram pressure compression might lead to an enhanced total power emission and a flattening of the radio continuum spectrum. However, based on integrated galaxy properties, Niklas (1995) and Vollmer et al. (2004a,b) do not find any significant difference between the mean spectral index of Virgo cluster and field spiral galaxies.

It is still an open question if environmental interactions in a galaxy cluster can locally enhance the total power radio continuum emission of a galaxy and if they can alter the spectral index as suggested by Vollmer et al. (2004a,b) for NGC 4522 in the Virgo cluster.

In this article we present the total power radio continuum observations of 8 Virgo spiral galaxies observed by Vollmer et al. (2007) in polarization. These galaxies were carefully selected based on the following criteria: (i) they show signs of interaction with the cluster environment such as tidal interactions and/or ram pressure stripping; (ii) VIVA HI data (Chung et al. 2009) are available; and (iii) their $6 \mathrm{~cm}$ total power emission is strong. In previous work we elaborated interaction scenarios for most of the galaxies in our sample.

Deep VLA 20 and $6 \mathrm{~cm}$ observations including polarization are presented in Sect. 2. In Sect. 3 we present for each galaxy the (i) $6 \mathrm{~cm}$ total power emission distribution superposed on DSS $B$ band image together with the apparent $\boldsymbol{B}$ vectors; (ii) $20 \mathrm{~cm}$ total power emission distribution on a DSS $B$ band image together with the apparent $\boldsymbol{B}$ vectors; (iii) $6 \mathrm{~cm}$ polarized emission distribution on the HI distribution; (iv) $\mathrm{H} \alpha$ emission distribution (from Goldmine et al. 2003) on the spectral index map; and (v) $6 \mathrm{~cm}$ polarized emission distribution on the degree of polarization. The galaxy properties at different wavelengths are compared to each other in Sect. 4. We discuss our results in Sect. 5 and give our conclusions in Sect. 6.

\section{Observations}

The 8 Virgo spiral galaxies were observed at $4.85 \mathrm{GHz}$ between November 8, 2005 and January 10, 2006 with the Very Large
Array (VLA) of the National Radio Astronomy Observatory $(\mathrm{NRAO})^{1}$ in the $\mathrm{D}$ array configuration. The band passes were $2 \times 50 \mathrm{MHz}$. We used 3C286 as the flux calibrator and 1254+116 as the phase calibrator, the latter of which was observed every $40 \mathrm{~min}$. Maps were made for both wavelengths using the AIPS task IMAGR with ROBUST $=3$. The final cleaned maps were convolved to a beam size of $18^{\prime \prime} \times 18^{\prime \prime}$. The bright radio source M 87 caused sidelobe effects enhancing the rms noise level of NGC 4438. In addition, we observed the 8 galaxies at $1.4 \mathrm{GHz}$ on August 15, 2005 in the $\mathrm{C}$ array configuration. The band passes were $2 \times 50 \mathrm{MHz}$. We used the same calibrators as for the $4.85 \mathrm{GHz}$ observations. The final cleaned maps were convolved to a beam size of $20^{\prime \prime} \times 20^{\prime \prime}$. The rms levels of the 20 and $6 \mathrm{~cm}$ total power and polarized intensity data are shown in Table 1 . We obtain apparent $\boldsymbol{B}$ vectors by rotating the observed $\boldsymbol{E}$ vector by $90^{\circ}$, uncorrected for Faraday rotation.

For NGC 4388, NGC 4402, NGC 4438, and NGC 4501 our $20 \mathrm{~cm}$ total power data have artifacts most likely due to the tenuous UV coverage. We therefore preferred the VIVA HI $20 \mathrm{~cm}$ continuum images (Chung et al. 2009; for the more sophisticated data reduction of NGC 4438 see Vollmer et al. 2009).

\section{Results}

\subsection{Galaxy properties}

\subsubsection{NGC 4321}

NGC 4321 is the only galaxy in our sample which shows relatively symmetric emission distributions at all wavelengths as it is observed in field spiral galaxies (Fig. 1). The $6 \mathrm{~cm}$ and $20 \mathrm{~cm}$ total power distributions follow the stellar and gas distribution. The radio continuum spectrum is flat $\left(\alpha>-0.8\right.$ with $\left.S_{v} \propto v^{\alpha}\right)$ in the regions of the main spiral arms and steep $(\alpha \leq-0.8)$ elsewhere. The polarized emission and the degree of polarization are highest in the interarm regions. In addition, the distribution of the degree of polarization shows an azimuthally symmetric radial gradient with a higher degree of polarization towards the outer disk. All characteristics cited above are typical for an unperturbed spiral galaxy (Beck 2005, and references therein). The regions of highest degrees of polarization (up to 40\%) are found at the western edge of the polarized emission distribution.

\footnotetext{
1 NRAO is a facility of National Science Foundation operated under cooperative agreement by Associated Universities, Inc.
} 
B. Vollmer et al.: Large-scale radio continuum emission of 8 Virgo cluster spirals
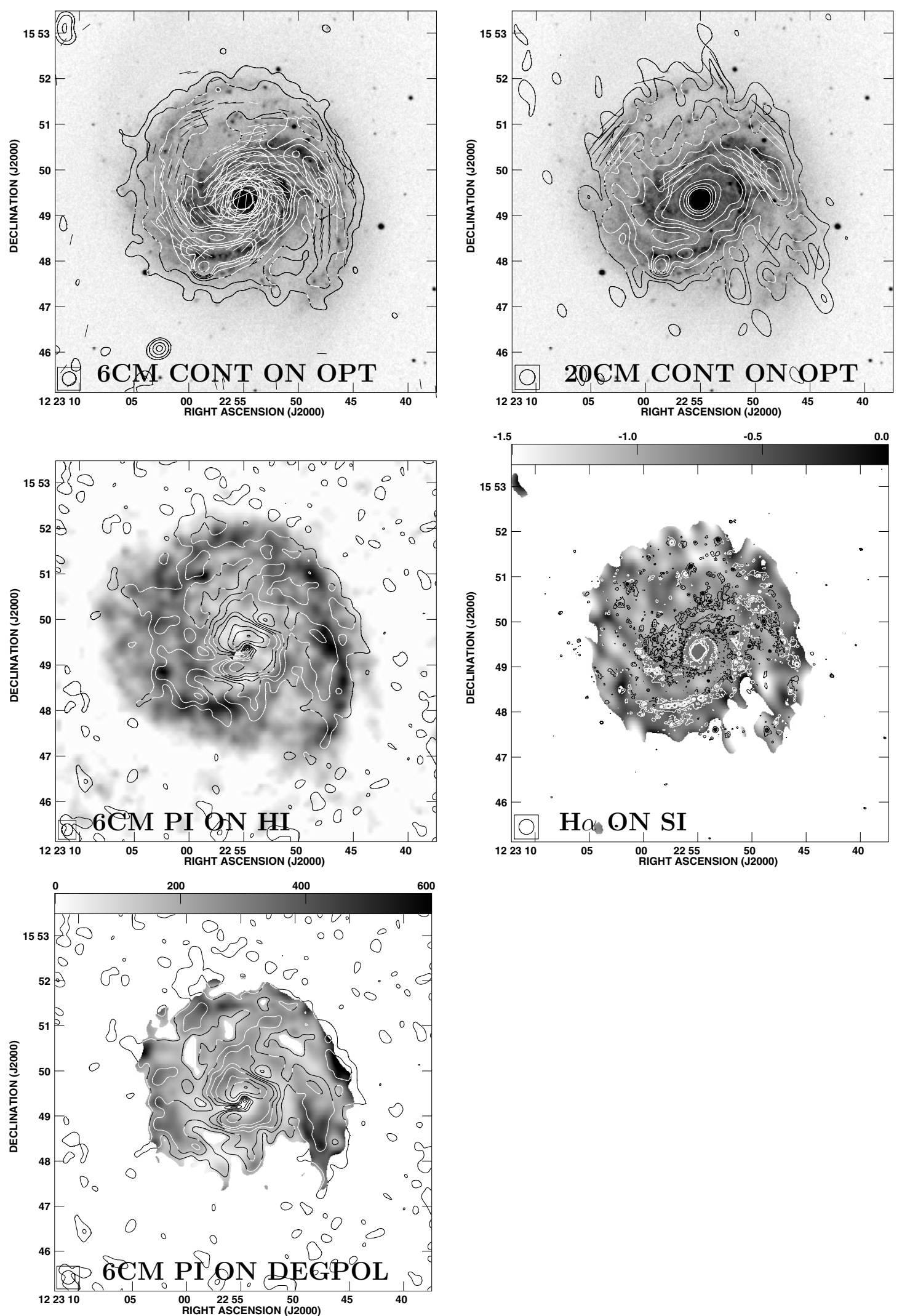

Fig. 1. NGC 4321: From top left to bottom right: $6 \mathrm{~cm}$ total power emission distribution on DSS $B$ band image together with the apparent $\boldsymbol{B}$ vectors, $20 \mathrm{~cm}$ total power emission distribution on DSS $B$ band image together with the apparent $\boldsymbol{B}$ vectors, $6 \mathrm{~cm}$ polarized emission distribution on VIVA HI distribution, $\mathrm{H} \alpha$ emission distribution on spectral index map, and $6 \mathrm{~cm}$ polarized emission distribution on degree of polarization. $6 \mathrm{~cm}$ continuum contour levels are $70 \mu \mathrm{Jy} \times(1,2,4,6,8,10,20,30,40,50) .20 \mathrm{~cm}$ continuum contour levels are $330 \mu \mathrm{Jy} \times(1,2,4,6,8,10,20,30,40$, 50). $6 \mathrm{~cm}$ polarized intensity contour levels are $10 \mu \mathrm{Jy} \times(4,8,12,16,20,30,40,50)$. The $\mathrm{H} \alpha$ contours on the spectral index map appear white if the spectral index $\alpha>-0.8$ where $S \propto v^{\alpha}$. The indicated levels of the degree of polarization are in units of $0.1 \%$. 

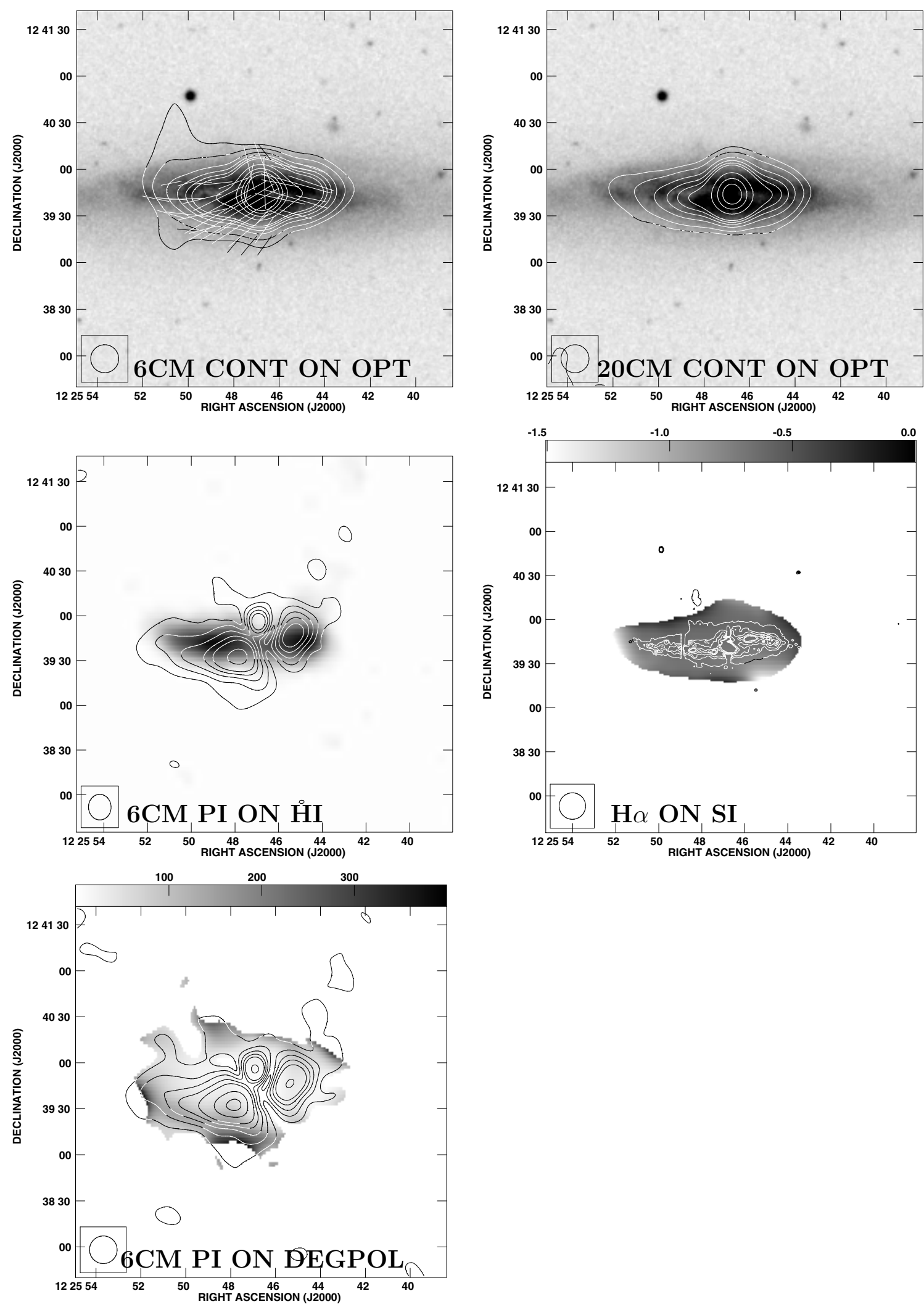

Fig. 2. NGC 4388: From top left to bottom right: $6 \mathrm{~cm}$ total power emission distribution on DSS $B$ band image together with the apparent $\boldsymbol{B}$ vectors, $20 \mathrm{~cm}$ total power emission distribution on DSS $B$ band image together with the apparent $\boldsymbol{B}$ vectors, $6 \mathrm{~cm}$ polarized emission distribution on VIVA $\mathrm{HI}$ distribution, $\mathrm{H} \alpha$ emission distribution on spectral index map, and $6 \mathrm{~cm}$ polarized emission distribution on degree of polarization. $6 \mathrm{~cm}$ continuum contour levels are $320 \mu \mathrm{Jy} \times(1,2,4,6,8,10,20,30,40,50) .20 \mathrm{~cm}$ continuum contour levels are $1500 \mu \mathrm{Jy} \times(1,2,4,6,8,10,20,30,40,50)$. $6 \mathrm{~cm}$ polarized intensity contour levels are $10 \mu \mathrm{Jy} \times(4,8,12,16,20,30,40,50)$. The $\mathrm{H} \alpha$ contours on the spectral index map appear white if the spectral index $\alpha>-0.8$ where $S \propto v^{\alpha}$. The indicated levels of the degree of polarization are in units of $0.1 \%$. 
Table 2. Integrated flux densities.

\begin{tabular}{lrrrr}
\hline \hline Galaxy name & $\begin{array}{r}S_{\mathrm{TP20} \mathrm{cm}} \\
\mathrm{mJy}\end{array}$ & $\begin{array}{r}S_{\text {PI20 cm }} \\
\mathrm{mJy}\end{array}$ & $\begin{array}{r}S_{\mathrm{TP} 6 \mathrm{~cm}} \\
\mathrm{mJy}\end{array}$ & $\begin{array}{r}S_{\text {PI6 cm }} \\
\mathrm{mJy}\end{array}$ \\
\hline NGC 4321 & $256 \pm 2$ & $17.2 \pm 0.5$ & $99.1 \pm 0.2$ & $14.1 \pm 0.2$ \\
NGC 4388 & $178 \pm 2$ & - & $81.6 \pm 0.5$ & $2.3 \pm 0.1$ \\
NGC 4396 & $15.8 \pm 0.5$ & $1.5 \pm 0.2$ & $8.3 \pm 0.1$ & $1.0 \pm 0.1$ \\
NGC 4402 & $68 \pm 2$ & - & $26.3 \pm 0.2$ & $2.5 \pm 0.1$ \\
NGC 4438 & $102 \pm 3$ & - & $73.9 \pm 0.3$ & $6.3 \pm 0.1$ \\
NGC 4501 & $331 \pm 2$ & $10.4 \pm 0.4$ & $96.2 \pm 0.2$ & $11.2 \pm 0.1$ \\
NGC 4535 & $66 \pm 1$ & $13.9 \pm 0.4$ & $28.7 \pm 0.2$ & $4.2 \pm 0.1$ \\
NGC 4654 & $134.6 \pm 0.5$ & $6.0 \pm 0.3$ & $49.9 \pm 0.1$ & $3.1 \pm 0.1$ \\
\hline
\end{tabular}

\subsubsection{NGC 4388}

NGC 4388 harbors a Seyfert 2 nucleus with an associated bright radio source and a $15^{\prime \prime}$-long jet to the north (Hummel \& Saikia 1991). The disk and jet emission perpendicular to the galactic plane has already been detected at $6 \mathrm{~cm}$ by Hummel et al. (1983). In Fig. 2 we observe emission from the nuclear outflow in polarized $6 \mathrm{~cm}$ emission with vertical magnetic field vectors north of the nucleus. The radio continuum disk emission at $20 \mathrm{~cm}$ and $6 \mathrm{~cm}$ and the HI emission distribution are truncated at about half the optical radius. These emission distributions are more extended to the east than to the west. The $6 \mathrm{~cm}$ total power extension perpendicular to the major axis east of the nucleus needs confirmation. It is present in observations made on two separate days. We do not detect a disk-wide radio halo. The eastern $6 \mathrm{~cm}$ polarized emission maximum is located $\sim 20^{\prime \prime}$ south of the disk plane, on the edge of the HI emission. The western $6 \mathrm{~cm}$ polarized emission maximum is slightly elongated parallel to the minor axis. The radio continuum spectrum is flat $(\alpha>-0.8)$ everywhere in the disk. We observe an asymmetric distribution of the degree of polarization at $6 \mathrm{~cm}$ with $10 \%$ of polarization at the southeastern edge, whereas the degree of polarization is much less at the northern edge of the galactic disk.

\subsubsection{NGC 4396}

The $20 \mathrm{~cm}$ and $6 \mathrm{~cm}$ total power emission of NGC 4396 is truncated within the optical disk and shows a tail structure to the northwest (Fig. 3). As the HI tail, the radio continuum tail is bend to the north, but it is less extended than the HI tail. We do not detect a disk-wide radio halo. The $6 \mathrm{~cm}$ polarized emission is mainly found in the southeast of the galactic disk extending into the tail. Faint polarized emission perpendicular to the galactic disk is found in the eastern region. The radio continuum spectrum is flat over the whole starforming disk of NGC 4396. The distribution of the degree of polarization at $6 \mathrm{~cm}$ increases towards the tail with a maximum value of $30 \%$ at the northwestern tip. It also increases to the north in the eastern polarized emission feature.

\subsubsection{NGC 4402}

As the HI emission distribution, the $20 \mathrm{~cm}$ and $6 \mathrm{~cm}$ total power emission distribution are truncated at about half of the optical radius (Chung et al. 2009, Fig. 4). In addition, we observe a disk-wide extension to the north, whereas the southern edge of the emission distributions is sharp (see also Crowl et al. 2005). Although the total power disk emission is symmetric along the major axis, the $6 \mathrm{~cm}$ polarized emission is more extended to the west than to the east. Moreover, we find faint extraplanar polarized emission northeast of the galactic disk. The extended extraplanar radio continuum emission north of the disk shows a steepening of the radio continuum spectrum. The distribution of the degree of polarization has a north-south and an east-west gradient, the latter being dominant. At the southern edge the degree of polarization is $10 \%$, whereas it increases up to $40 \%$ at the western edge of the galactic disk.

\subsubsection{NGC 4438}

NGC 4438 harbors a Seyfert 2 nucleus with an associated strong nuclear radio source (Hummel \& Saikia 1991). In addition, we observe prominent extraplanar total power emission at $20 \mathrm{~cm}$ and $6 \mathrm{~cm}$ extending further than emission at other wavelengths (Fig. 5). The $6 \mathrm{~cm}$ polarized extraplanar emission has a shell-like distribution with a pronounced maximum in the south. We also observe polarized emission from the galactic disk south from the nucleus. The spectral index of the western extraplanar emission region of NGC 4438 is constant. Its value is uncertain because of M 87's strong sidelobes at $20 \mathrm{~cm}$ which makes the $20 \mathrm{~cm}$ flux density uncertain. In Vollmer et al. (2009) we argue that we miss a substantial part of the flux density of NGC 4438 at $20 \mathrm{~cm}$ due to these sidelobes and that the spectral index is most likely $\alpha=-0.8$, i.e. the typical value for synchrotron emission. There is a positive gradient of the degree of polarization towards the border of the extraplanar synchrotron emission where the degree of polarization increases to $\sim 30 \%$. The southern half of the extraplanar radio emission shows a degree of polarization of $20 \%$.

\subsubsection{NGC 4501}

As the HI distribution, the $20 \mathrm{~cm}$ and $6 \mathrm{~cm}$ total power emission distributions are truncated at about the optical radius (Fig. 6). Whereas the southwestern edge is sharp, there is extended emission to the northeast. The $6 \mathrm{~cm}$ polarized emission shows a long ridge extending over almost the entire south western edge of the galactic disk. There is a secondary maximum north of the nucleus. Whereas the radio continuum spectrum is flat $(\alpha>-0.8)$ in the northeastern spiral arm, it is steep $(\alpha \leq-0.8)$ in the southwestern spiral arms. We observe a general steepening of the spectrum to the southeast of the galactic disk. The degree of polarization shows an asymmetric distribution. It increases towards the southwestern and northeastern edges of the galactic disk. Whereas it rises to $20 \%$ in the northeast, it increases to $30 \%$ in the southwest.

\subsubsection{NGC 4535}

The $20 \mathrm{~cm}$ and $6 \mathrm{~cm}$ total power emission extends to $\sim 0.7$ times the optical radius, well inside the HI distribution (Fig. 7). The total power emission associated with the western optical arm is stronger than that of the rest of the disk. Polarized $6 \mathrm{~cm}$ continuum emission is only detected in the region of the western optical arm. The radio continuum spectrum is flat $(\alpha>-0.8)$ in the regions of the starforming spiral arms and steep $(\alpha \leq-0.8)$ elsewhere. A high degree of polarization $(\sim 40 \%)$ is found in the southern part of the polarized emission arm. 
A\&A 512, A36 (2010)
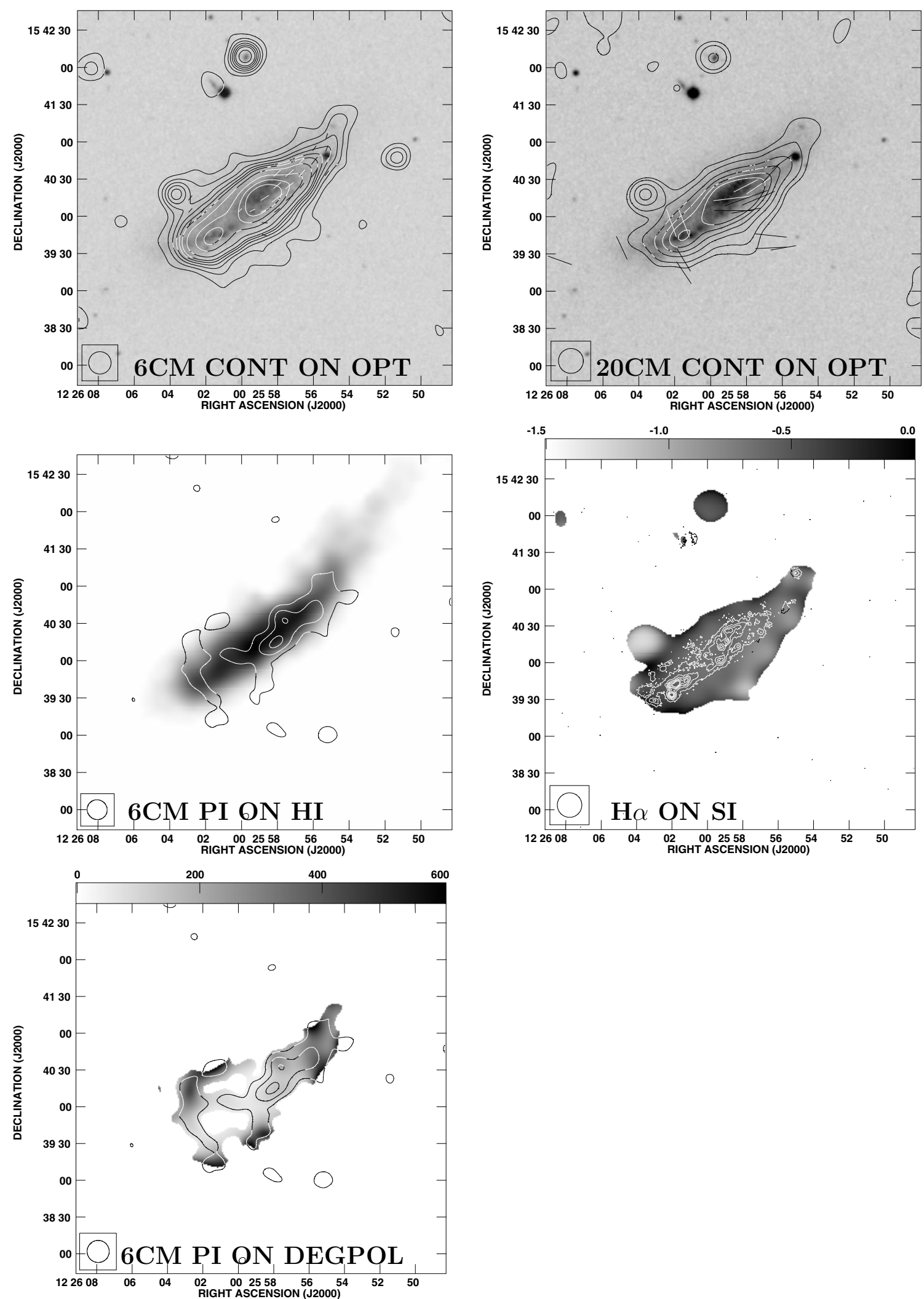

Fig. 3. NGC 4396: From top left to bottom right: $6 \mathrm{~cm}$ total power emission distribution on DSS $B$ band image together with the apparent $\boldsymbol{B}$ vectors, $20 \mathrm{~cm}$ total power emission distribution on DSS $B$ band image together with the apparent $\boldsymbol{B}$ vectors, $6 \mathrm{~cm}$ polarized emission distribution on VIVA $\mathrm{HI}$ distribution, $\mathrm{H} \alpha$ emission distribution on spectral index map, and $6 \mathrm{~cm}$ polarized emission distribution on degree of polarization. $6 \mathrm{~cm}$ continuum contour levels are $40 \mu \mathrm{Jy} \times(1,2,3,4,5,6,8,12,16,20,30,40,50) .20 \mathrm{~cm}$ continuum contour levels are $150 \mu \mathrm{Jy} \times(1,2,4,6,8,10,20,30,40$, 50). $6 \mathrm{~cm}$ polarized intensity contour levels are $8 \mu \mathrm{Jy} \times(4,8,12,16,20,30,40,50)$. The $\mathrm{H} \alpha$ contours on the spectral index map appear white if the spectral index $\alpha>-0.8$ where $S \propto v^{\alpha}$. The indicated levels of the degree of polarization are in units of $0.1 \%$. 
B. Vollmer et al.: Large-scale radio continuum emission of 8 Virgo cluster spirals
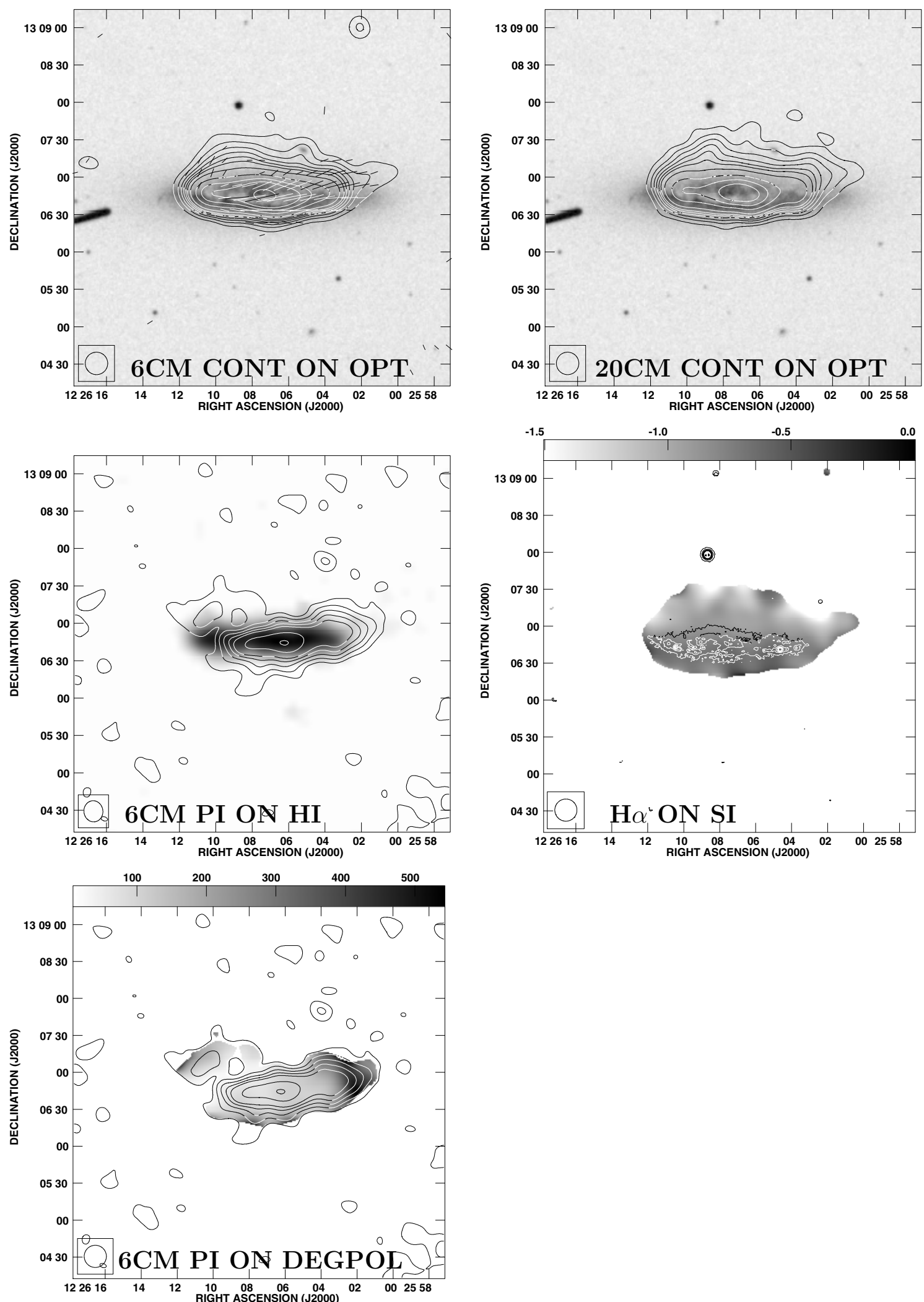

Fig. 4. NGC 4402: From top left to bottom right: $6 \mathrm{~cm}$ total power emission distribution on DSS $B$ band image together with the apparent $\boldsymbol{B}$ vectors, $20 \mathrm{~cm}$ total power emission distribution on DSS $B$ band image together with the apparent $\boldsymbol{B}$ vectors, $6 \mathrm{~cm}$ polarized emission distribution on VIVA $\mathrm{HI}$ distribution, $\mathrm{H} \alpha$ emission distribution on spectral index map, and $6 \mathrm{~cm}$ polarized emission distribution on degree of polarization. $6 \mathrm{~cm}$ continuum contour levels are $120 \mu \mathrm{Jy} \times(1,2,3,4,5,6,8,12,16,20,30,40,50) .20 \mathrm{~cm}$ continuum contour levels are $400 \mu \mathrm{Jy} \times(1,2,3,4,5,6,8,12,16,20$, $30,40,50) .6 \mathrm{~cm}$ polarized intensity contour levels are $8 \mu \mathrm{Jy} \times(4,8,12,16,20,30,40,50)$. The $\mathrm{H} \alpha$ contours on the spectral index map appear white if the spectral index $\alpha>-0.8$ where $S \propto v^{\alpha}$. The indicated levels of the degree of polarization are in units of $0.1 \%$. 

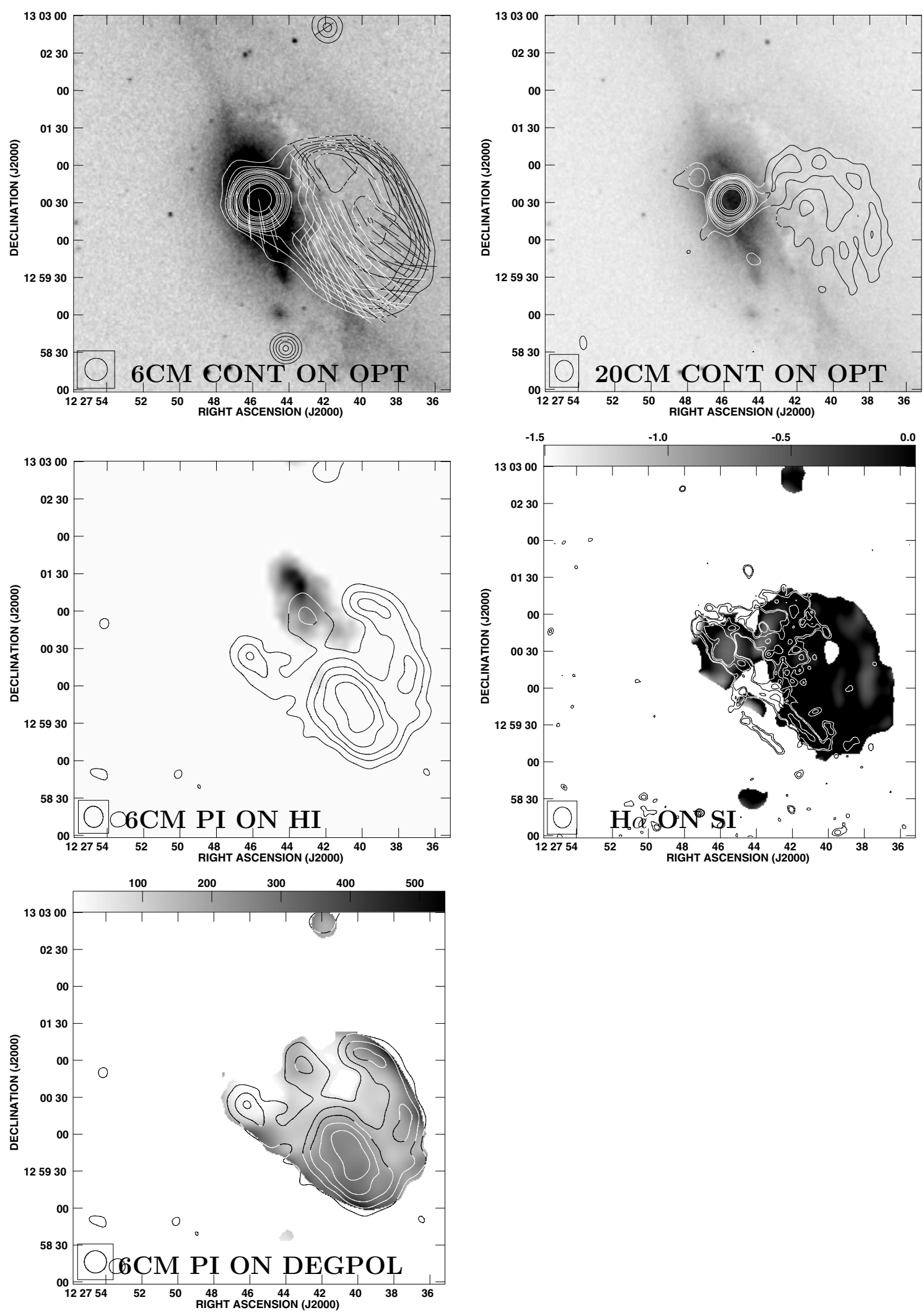

Fig. 5. NGC 4438: From top left to bottom right: $6 \mathrm{~cm}$ total power emission distribution on DSS $B$ band image together with the apparent $\boldsymbol{B}$ vectors, $20 \mathrm{~cm}$ total power emission distribution on DSS $B$ band image together with the apparent $\boldsymbol{B}$ vectors, $6 \mathrm{~cm}$ polarized emission distribution on VIVA HI distribution, $\mathrm{H} \alpha$ emission distribution on spectral index map, and $6 \mathrm{~cm}$ polarized emission distribution on degree of polarization. $6 \mathrm{~cm}$ continuum contour levels are $160 \mu \mathrm{Jy} \times(1,2,3,4,5,6,8,12,16,20,30,40,50) .20 \mathrm{~cm}$ continuum contour levels are $180 \mu \mathrm{Jy} \times(1,2,3,4,5,6,8,12,16,20$, $30,40,50) .6 \mathrm{~cm}$ polarized intensity contour levels are $15 \mu \mathrm{Jy} \times(4,8,12,16,20,30,40,50)$. The $\mathrm{H} \alpha$ contours on the spectral index map appear white if the spectral index $\alpha>-0.8$ where $S \propto v^{\alpha}$. The indicated levels of the degree of polarization are in units of $0.1 \%$. 
B. Vollmer et al.: Large-scale radio continuum emission of 8 Virgo cluster spirals
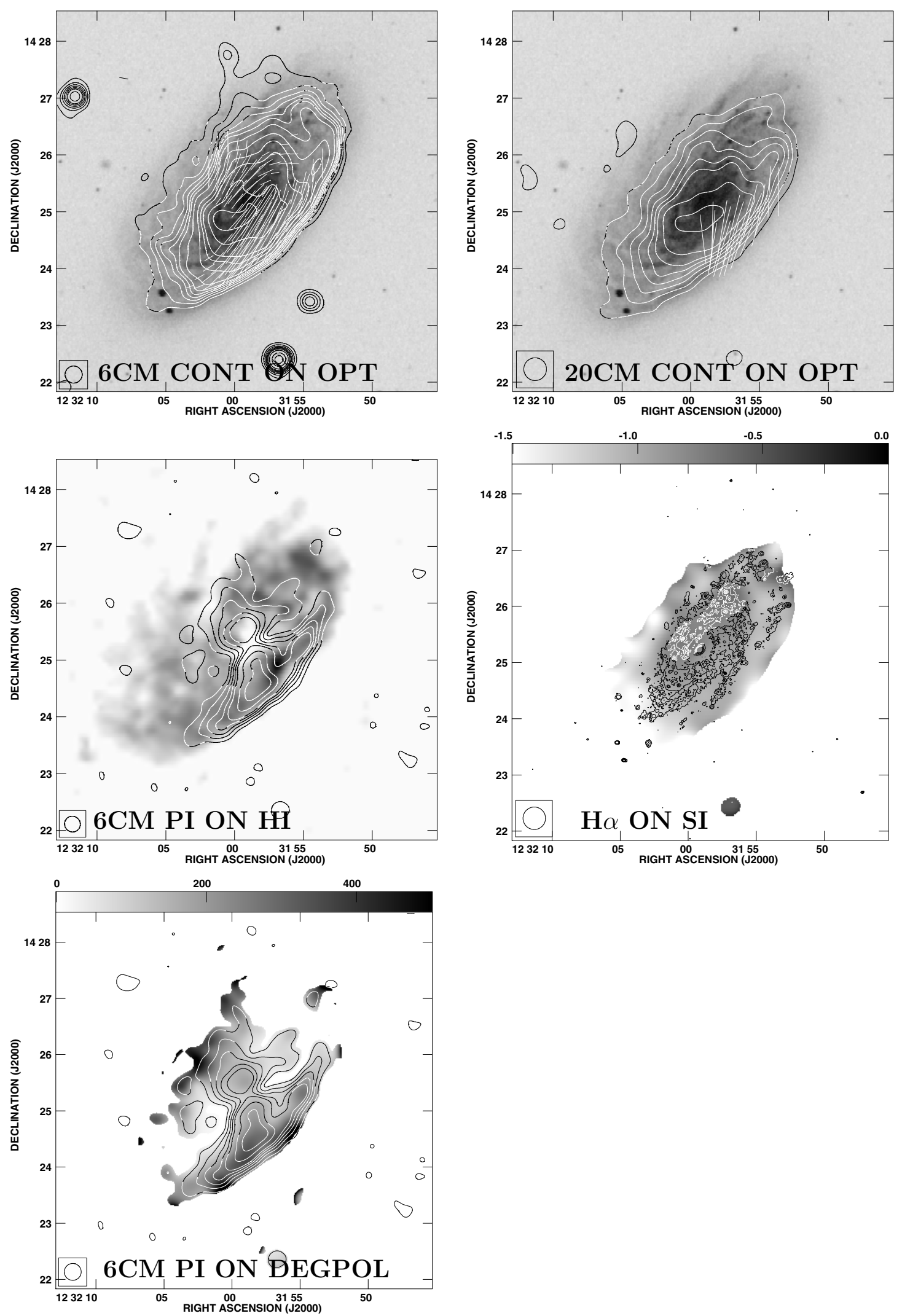

Fig. 6. NGC 4501: From top left to bottom right: $6 \mathrm{~cm}$ total power emission distribution on DSS $B$ band image together with the apparent $\boldsymbol{B}$ vectors, $20 \mathrm{~cm}$ total power emission distribution on DSS $B$ band image together with the apparent $\boldsymbol{B}$ vectors, $6 \mathrm{~cm}$ polarized emission distribution on VIVA HI distribution, $\mathrm{H} \alpha$ emission distribution on spectral index map, and $6 \mathrm{~cm}$ polarized emission distribution on degree of polarization. $6 \mathrm{~cm}$ continuum contour levels are $80 \mu \mathrm{Jy} \times(1,2,3,4,5,6,8,12,16,20,30,40,50) .20 \mathrm{~cm}$ continuum contour levels are $1500 \mu \mathrm{Jy} \times(1,2,3,4,5,6,8,12,16,20$, $30,40,50) .6 \mathrm{~cm}$ polarized intensity contour levels are $15 \mu \mathrm{Jy} \times(4,8,12,16,20,30,40,50)$. The $\mathrm{H} \alpha$ contours on the spectral index map appear white if the spectral index $\alpha>-0.8$ where $S \propto v^{\alpha}$. The indicated levels of the degree of polarization are in units of $0.1 \%$. 

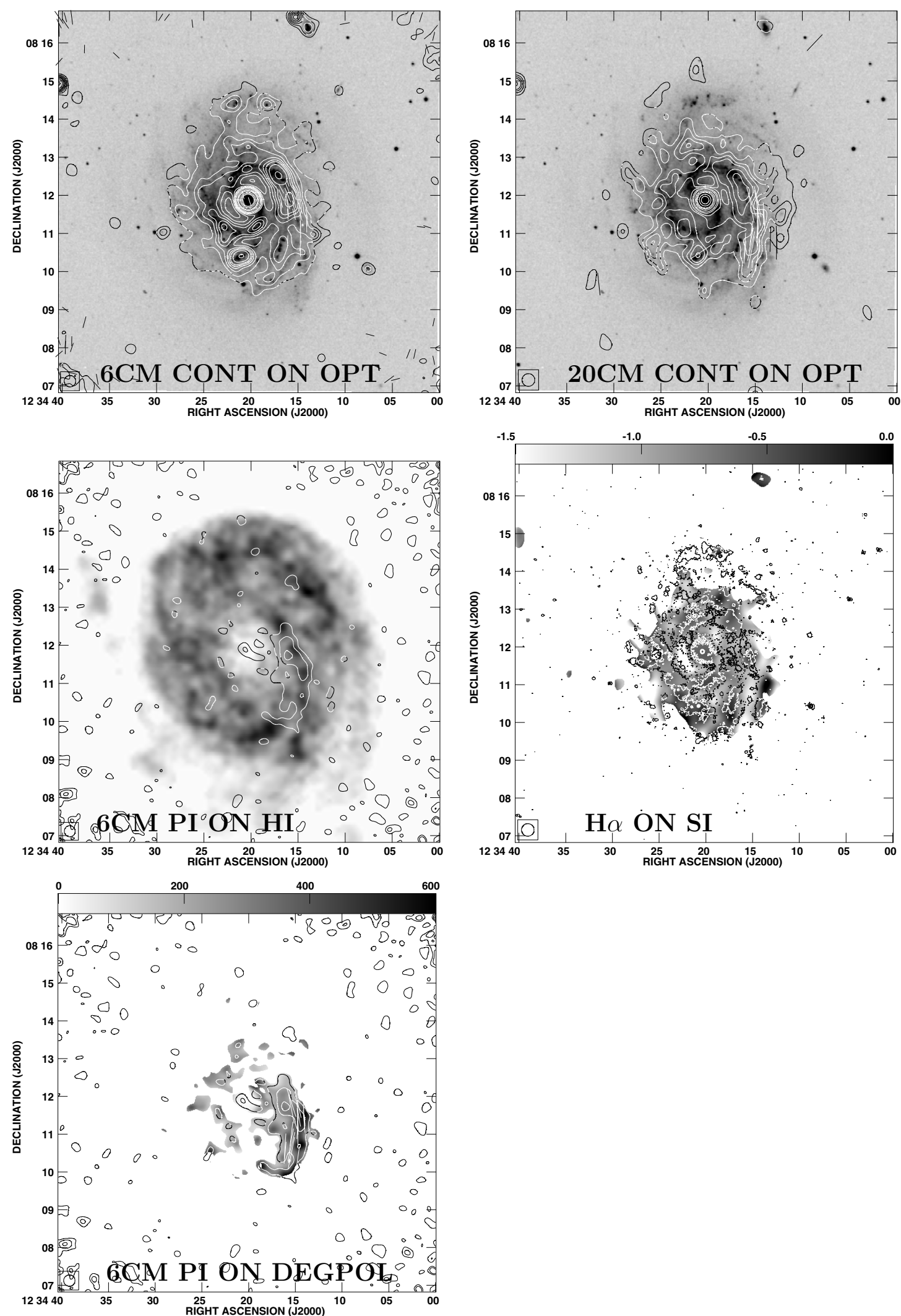

Fig. 7. NGC 4535: From top left to bottom right: $6 \mathrm{~cm}$ total power emission distribution on DSS $B$ band image together with the apparent $\boldsymbol{B}$ vectors, $20 \mathrm{~cm}$ total power emission distribution on DSS $B$ band image together with the apparent $\boldsymbol{B}$ vectors, $6 \mathrm{~cm}$ polarized emission distribution on VIVA HI distribution, $\mathrm{H} \alpha$ emission distribution on spectral index map, and $6 \mathrm{~cm}$ polarized emission distribution on degree of polarization. $6 \mathrm{~cm}$ continuum contour levels are $55 \mu \mathrm{Jy} \times(1,2,3,4,5,6,8,12,16,20,30,40,50) .20 \mathrm{~cm}$ continuum contour levels are $220 \mu \mathrm{Jy} \times(1,2,3,4,6,8,12,16,20$, $30,40,50) .6 \mathrm{~cm}$ polarized intensity contour levels are $10 \mu \mathrm{Jy} \times(4,8,12,16,20,30,40,50)$. The $\mathrm{H} \alpha$ contours on the spectral index map appear white if the spectral index $\alpha>-0.8$ where $S \propto v^{\alpha}$. The indicated levels of the degree of polarization are in units of $0.1 \%$. 
B. Vollmer et al.: Large-scale radio continuum emission of 8 Virgo cluster spirals
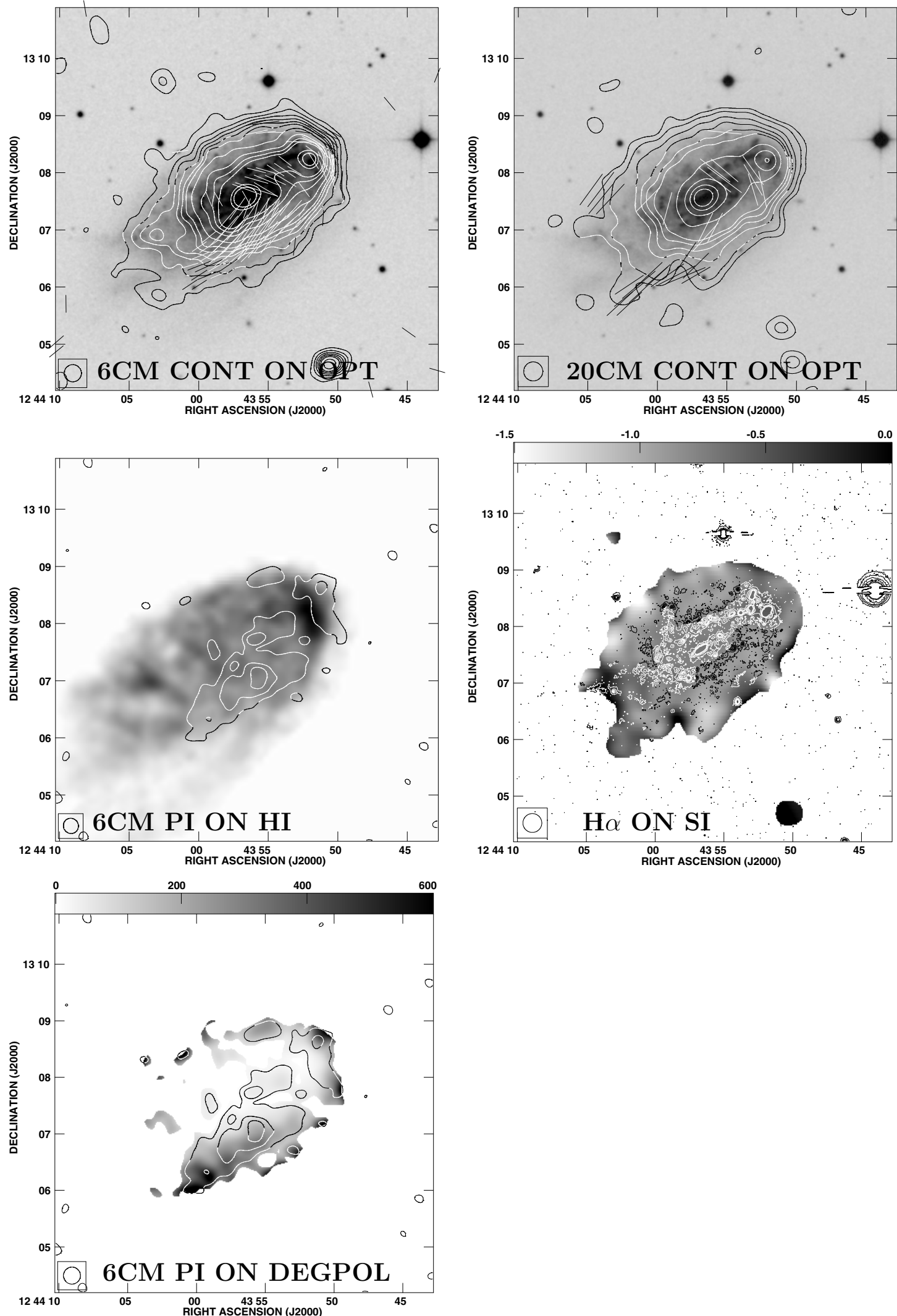

Fig. 8. NGC 4654: From top left to bottom right: $6 \mathrm{~cm}$ total power emission distribution on DSS $B$ band image together with the apparent $\boldsymbol{B}$ vectors, $20 \mathrm{~cm}$ total power emission distribution on DSS $B$ band image together with the apparent $\boldsymbol{B}$ vectors, $6 \mathrm{~cm}$ polarized emission distribution on VIVA $\mathrm{HI}$ distribution, $\mathrm{H} \alpha$ emission distribution on spectral index map, and $6 \mathrm{~cm}$ polarized emission distribution on degree of polarization. $6 \mathrm{~cm}$ continuum contour levels are $57 \mu \mathrm{Jy} \times(1,2,4,6,8,10,20,30,40,50) .20 \mathrm{~cm}$ continuum contour levels are $180 \mu \mathrm{Jy} \times(1,2,3,4,6,8,12,16,20,30,40,50)$. $6 \mathrm{~cm}$ polarized intensity contour levels are $10 \mu \mathrm{Jy} \times(4,8,12,16,20,30,40,50)$. Optical DSS and VIVA HI images are used. The H $\alpha$ contours on the spectral index map appear white if the spectral index $\alpha>-0.8$ where $S \propto v^{\alpha}$. The indicated levels of the degree of polarization are in units of $0.1 \%$. 


\subsubsection{NGC 4654}

The $20 \mathrm{~cm}$ and $6 \mathrm{~cm}$ total power emission roughly follows the stellar distribution of the galactic disk (Fig. 8). Along the major axis, the emission is stronger to the northwest than to the southeast. We observe a sharp edge of the emission distribution to the northwest and some faint extended emission in the direction of the prominent southeastern HI tail. The $6 \mathrm{~cm}$ polarized emission distribution is asymmetric with a maximum south of the galaxy center well within the HI distribution. The radio continuum spectrum is flat $(\alpha>-0.8)$ in the regions of the starforming spiral arms and steep $(\alpha \leq-0.8)$ elsewhere. The degree of $6 \mathrm{~cm}$ polarization increase to $\sim 20 \%$ towards the southern edge of the polarized emission distribution. The highest degrees of polarization $(\sim 30 \%)$ are found at the south eastern tip.

\section{Comparison between galaxies}

In this section we compare the total power distributions at $20 / 6 \mathrm{~cm}$ to the gas and stellar disks followed by the polarized radio continuum emission and the spectral index. The degree of polarization at $6 \mathrm{~cm}$ and the rotation measure between 20 and $6 \mathrm{~cm}$ are presented at the end of this section.

\subsection{Total power emission}

The total power emission is a mixture of synchrotron and thermal emission. The intensity of the synchrotron emission depends on the density of cosmic-ray electrons and the square of the total magnetic field in the sky plane. The density of thermal and of cosmic ray electrons depends on the star formation activity of the galaxy. In an isolated spiral galaxy the total magnetic field is dominated by the turbulent small-scale component. As the result of ram pressure compression the total power emission can be locally enhanced by (i) an increased local star formation rate and/or (ii) compression of the magnetic field.

Only NGC 4321 shows a symmetric total power distribution as it is observed in unperturbed field spiral galaxies. The $20 \mathrm{~cm}$ and $6 \mathrm{~cm}$ total power distributions of two (NGC 4501, NGC 4654) out of three mildly inclined spiral galaxies show sharp edges with steep gradients at one side of the disk. These edges coincide with HI edges and are due to ram pressure (Soida et al. 2006; Vollmer et al. 2008). Murphy et al. (2009) combined Spitzer FIR and VLA $20 \mathrm{~cm}$ radio continuum imaging to study the FIR-radio correlation in Virgo spiral galaxies. For 6 out of 10 sample galaxies, they found regions along their outer edges that are highly deficient in the radio compared with models relying on the FIR-radio correlation of field galaxies. The observed sharp edges in the total power distribution might be linked to this phenomenon of radio deficient edges.

We observe asymmetric extraplanar emission in 3 out of 4 edge-on galaxies (NGC 4396, NGC 4402, NGC 4438; see Sect. 5.1). Whereas the extraplanar radio continuum emission follows the HI emission in the northwest of NGC 4396, it is more extended than the HI emission in NGC 4402 and NGC 4438. At our limiting flux density level there is no total power emission associated with the ionized nuclear outflow in NGC 4388 (Veilleux et al. 1999). In all moderately inclined galaxies (NGC 4321, NGC 4501, NGC 4535, NGC 4654) the $20 \mathrm{~cm}$ and $6 \mathrm{~cm}$ emission follow the recent massive star formation as observed in the $\mathrm{H} \alpha$ line. The southeastern HI tail of NGC 4654 (Phookun \& Mundy 1995) has no radio continuum counterpart. This is most probably due to the lack of star formation in this tail.

\subsection{Polarized continuum and Hi emission}

Polarized continuum emission is caused by the regular largescale magnetic field. Polarized emission can be enhanced by large-scale shear or compression motions.

Only NGC 4321 has a relatively symmetric polarized emission distribution which is highest in the interarm regions. All other galaxies have asymmetric elongated ridges of polarized emission located in the outer parts of the galactic disks (Vollmer et al. 2007). These ridges are located within the HI distribution, except for NGC 4438, where the western local maximum of polarized emission is located within the molecular gas disk (Vollmer et al. 2005). Most of the ridges are close to the outer edge of the HI distribution. Especially in NGC 4501 the HI and polarized emission distributions show a sharp edge to the southeast where ram pressure acts on the galaxy (see Vollmer et al. 2008). Such a sharp edge is also observed in the northeast of NGC 4654 (see Soida et al. 2006). Only the southern polarized ridge of NGC 4654 and that of NGC 4535 are well inside the HI distribution. Given the asymmetric velocity field of NGC 4535 in the region of the maximum of polarized radio continuum emission (Chung et al. 2009), the enhancement of the polarized emission is most probably due to shear motions. Moreover, all edge-on galaxies show extraplanar polarized emission which extends further than the HI emission. In NGC 4388 this extraplanar polarized emission is probably due to the AGN outflow (Veilleux et al. 1999). In NGC 4396 it is located on the southeast and extends to the north. In NGC 4402 it is located in the western side of the disk where the action of ram pressure is maximum (Crowl et al. 2005). Whereas the extraplanar polarized emission is faint in NGC 4396 and NGC 4402, it is prominent in NGC 4438, extending further than emission at any other wavelength.

The polarized emission at $20 \mathrm{~cm}$ suffers severe Faraday rotation and depolarization, especially in edge-on galaxies. We will use this emission only to calculate the rotation measure in Sect. 4.5.

\subsection{Spectral index}

As a general trend, we find flat radio continuum spectra $(\alpha>$ -0.8 ) associated with regions of recent massive star formation, indicating an enhanced fraction of thermal electrons. This corresponds to the classical behavior of unperturbed spiral galaxies (e.g., Sukumar et al. 1987; Berkhuijsen et al. 2003; Tabatabaei et al. 2007a,b; Heesen et al. 2009). For example, in NGC 4396 the radio continuum spectrum of the northwestern extraplanar emission region is flat $(\alpha \sim-0.6)$, because of its active star formation. On the other hand, the extraplanar radio continuum emission north of NGC 4402 shows a steepening of the spectrum due to the aging of the relativistic electrons. This is consistent with the scenario of Crowl et al. (2005) where it is assumed that the radio halo is compressed on the southern side and pushed out of the galactic disk on the northern side. Surprisingly, the same steepening of the spectrum is seen on the southwestern side of NGC 4501 where ram pressure compresses the gas (Vollmer et al. 2008). This is contrary to what we found in NGC 4522 (Vollmer et al. 2004a,b) where we observed a spectral flattening in the compressed region. Thus, in our sample we do not observe a flattening of the spectrum in regions of enhanced polarized emission. A shock-induced reacceleration of relativistic electrons as proposed by Völk \& Xu (1994) is still a probable explanation for the flat spectrum associated to the ridge of polarized emission in NGC 4522. 
Table 3. Polarized intensity weighted mean values of the rotation measure distribution in $\mathrm{rad} / \mathrm{m}^{2}$.

\begin{tabular}{cccccc}
\hline \hline & $\mathrm{N} 4321$ & $\mathrm{~N} 4396$ & $\mathrm{~N} 4501$ & $\mathrm{~N} 4535$ & $\mathrm{~N} 4654$ \\
\hline$\langle\mathrm{RM}\rangle$ & -5 & -7 & +10 & 2 & $-8 / 2$ \\
\hline
\end{tabular}

\subsection{Degree of polarization}

The degree of polarization is defined as the ratio between polarized and total power (mostly synchrotron) emission. The degree of polarization is a measure for the fraction of the regularly oriented, large-scale magnetic field with respect to the total magnetic field.

In our galaxy sample the degree of polarization varies between 10 and $40 \%$. In the face-on galaxy NGC 4321 the degree of polarization is highest in the interarm regions as it is expected for an unperturbed galaxy. Moreover, we observe an azimuthally symmetric radial gradient as it is observed in field spirals (e.g., M 83: Neininger et al. 1993; NGC 6946: Ehle \& Beck 1993). As an example for an edge-on spiral galaxy, Dumke \& Krause (1998) combined $6 \mathrm{~cm}$ Effelsberg and VLA data of NGC 891 (optical radius $R_{25}=13.5^{\prime}$ ). They found a symmetrically increasing degree of polarization towards the edges of the emission distribution to $\sim 10 \%$.

In our sample, NGC 4388, NGC 4396, and NGC 4402 show vertically asymmetric gradients of the degree of polarization. In addition, NGC 4396, NGC 4402, NGC 4501, and NGC 4654 show azimuthally asymmetric distributions of the degree of polarization. The rising degree of polarization towards the tails in NGC 4396 and NGC 4654 might be due to magnetic field ordering or shear motions in the gas which is pulled away from or re-accreting onto the galactic disk. Thus 5 out of 8 sample galaxies show these asymmetries. This is different from the behavior of field spirals (Beck 2005 and references therein; e.g. Neininger 1992; Sukumar \& Allen 1991) and is thus most probably due to the interaction between the galaxy and the cluster environment.

\subsection{Rotation measure}

The orientation of polarization vectors is changed in a magnetic plasma by Faraday rotation that is proportional to the line-ofsight integral over the density of thermal electrons multiplied by the strength of the regular field component along the line of sight. Aligned anisotropic fields do not lead to Faraday rotation.

The rotation measure was calculated with polarization angle maps which were clipped at $3 \sigma$ in polarized emission. This leads to a maximum uncertainty of the rotation measure of $\sim 16 \mathrm{rad} / \mathrm{m}^{2}$. Since the $20 \mathrm{~cm}$ data suffer from severe Faraday depolarization, we detect significant polarized emission at $20 \mathrm{~cm}$ only in NGC 4321, NGC 4501, NGC 4396, NGC 4535, and NGC 4654. The intensity-weighted mean values of the rotation measure for our galaxies are presented in Table 3. Since the rotation measure is caused by the regular magnetic field component along the line-of-sight, we expect the rotation measure from an axisymmetric field to change sign from one side of the major axis to the other. This is the reason why we give two values for the NGC 4654 which is the only galaxy where we can significantly determine rotation measures on both sides of the minor axis. In general, all face-on galaxies show detectable rotation measures and we find rotation measures between -7 and $10 \mathrm{rad} / \mathrm{m}^{2}$. However, since the difference in frequency is large and Faraday depolarization can be strong at $20 \mathrm{~cm}$, one has to take these measurements with caution. The actual rotation measures are most probably much larger. For comparison, Weżgowiec et al. (2007) report rotation measures between 4.85 and $10.45 \mathrm{GHz}$ obtained from Effelsberg observations of $+14-+46 \mathrm{rad} / \mathrm{m}^{2}$ for NGC 4501 and $-57 /+60 \mathrm{rad} / \mathrm{m}^{2}$ for NGC 4654. Ultimately, we would like to observe these galaxies at $3 \mathrm{~cm}$ to produce more reliable rotation measure maps. These will help us to discriminate between the 2 creation scenarios of the polarized ridges: in the case of shear or compression of a random field, the resulting ordered field is anisotropic and cannot produce Faraday rotation. A detectable rotation measure would show regular large-scale fields and thus help to distinguish them from anisotropic ones. A high rotation measure in regions of the ridges of enhanced polarized emission might indicate that a preexisting large-scale field has been amplified by compression or shear.

\section{Discussion}

\subsection{Disk-wide radio halos}

Only NGC 4402 out of three edge-on galaxies shows a diskwide radio halo that is compressed on one side and extends to $\sim 3.7 \mathrm{kpc}$ above the disk plane on the other side (Crowl et al. 2005). With increasing distance from the galactic disk the total power surface brightness decreases and the radio continuum spectrum steepens due to the aging of relativistic electrons generated in the galactic disk. This halo shows extraplanar polarized emission at the eastern and western extremities and vertical magnetic field lines in the east, unlike classical X-structures (Beck 2005 and references therein; e.g. Tüllmann et al. 2000), supporting the scenario of a ram pressure pushed/stripped synchrotron halo.

Dahlem et al. (2006) showed that disk-wide radio halos exist in galaxies with a low mean mass surface density and a high mean energy input per unit surface area (Fig. 7 of Dahlem et al. 2006). To investigate if our edge-on spirals should host a radio halo, we calculated the mean stellar mass surface density using the $H$ band magnitudes and optical diameters from Goldmine. For the mean energy input per unit surface area we use the $20 \mathrm{~cm}$ flux densities and radial extents. For NGC 4388 we subtracted the emission from the active nucleus. Figure 9 shows the border between galaxies with and without radio halos together with the data points of our 3 edge-on galaxies. Based on this plot NGC 4388 and NGC 4402 are expected to show a disk-wide radio halo, but only NGC 4402 does. A radio halo probably exists in NGC 4388, but may have been missed due to the higher noise in our maps compared to NGC 4402. If this is not the case, the pressure of the intracluster medium, that confines the radio halo might be higher for NGC 4402 than for NGC 4388.

\subsection{Extraplanar radio continuum emission can extend further than the Hiemission}

In our sample of 8 Virgo spiral galaxies we have two examples of asymmetric extraplanar total power emission extending further than the HI emission: NGC 4402, and NGC 4438. For comparison, in NGC 891 the distribution of neutral hydrogen has a vertical extent comparable to that of the radio halo (Oosterloo et al. 2007). This is not the case for NGC 4402 (Fig. 10) where the radio halo is more extended to the north than the HI distribution.

The extraplanar radio continuum emission of NGC 4438, located up to $5 \mathrm{kpc}$ from the galactic disk, is unique in the sense 


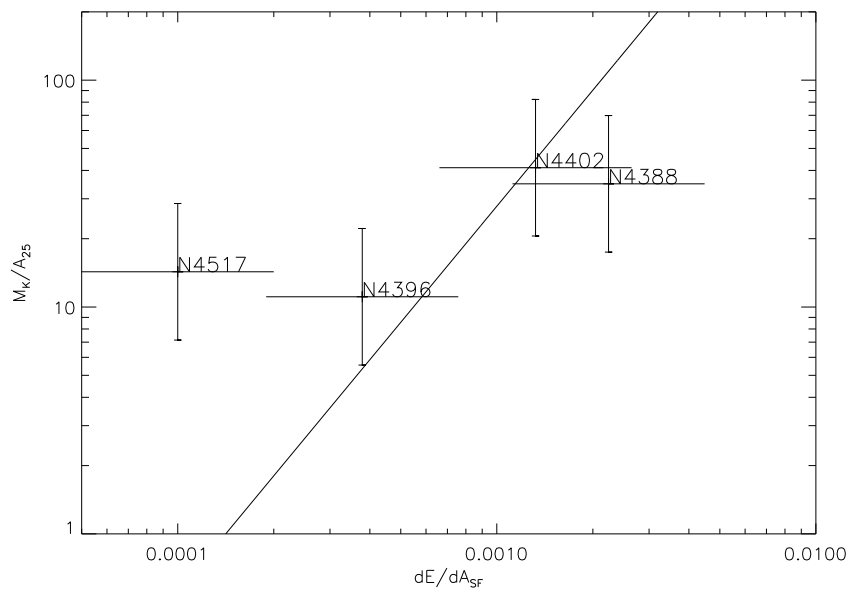

Fig. 9. Mean stellar mass density as a function of the mean energy input per unit surface area. The solid line roughly indicates the transition zone between galaxies with and without radio halos (Dahlem et al. 2006). The error bars represent uncertainties of a factor of 2 .

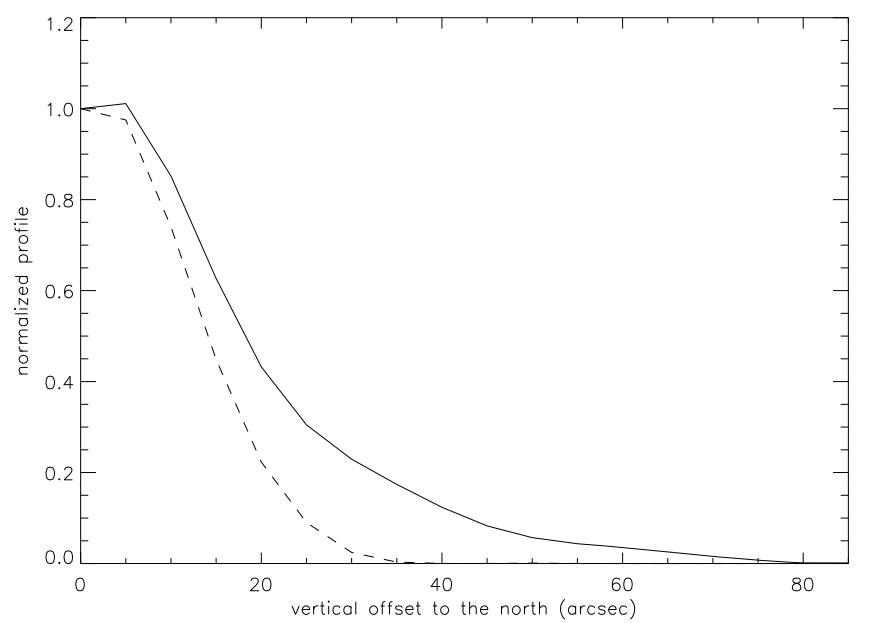

Fig. 10. NGC 4402: vertical profiles to the north of the $20 \mathrm{~cm}$ continuum (solid line) and HI (dashed line) emission distribution at a resolution of $20^{\prime \prime}$.

that it has an almost constant surface brightness and spectral index (Vollmer et al. 2009).

NGC 4396 and NGC 4402 show extraplanar $6 \mathrm{~cm}$ polarized emission in the southwestern (NGC 4396) and northeastern (NGC 4402) part of the disk extending $\sim 2.5 \mathrm{kpc}$ above the galactic plane. For NGC 4396 its association to the extraplanar total power emission is uncertain, because a relatively bright point source occupies the same region. In NGC 4402 this extraplanar polarized emission is associated with total power emission from the radio halo. NGC 4396 thus resembles NGC 4402, but it lacks a detectable radio halo, because of its low mean star formation rate (Fig. 9).

The radio continuum emission of the galaxies in our sample and in the literature (i) is more extended than the extraplanar $\mathrm{H} \alpha$ and $\mathrm{HI}$ emission in NGC 4402, NGC 4438, and NGC 4569 (Chyży et al. 2006); (ii) follows the $\mathrm{H} \alpha$ and $\mathrm{HI}$ emission in NGC 4501, and NGC 4522 (Vollmer et al. 2004a,b); and (iii) is less extended than the HI emission in NGC 4535 and NGC 4654. The asymmetric extraplanar radio continuum emission extending further than the HI emission may be due to (i) the ionization of ram pressure stripped HI; (ii) more efficient stripping of the cosmic ray gas with its associated magnetic field, or (iii) nuclear outflows.

\subsection{Ram pressure compression does not change the local total power emission nor the spectral index}

In all galaxies with asymmetric polarized emission ridges we do not detect significant enhanced total power emission associated with these ridges (Sect. 4.1). This means that the local star formation and the small-scale turbulent magnetic field, which is sensitive to star formation in unperturbed galaxies, are not affected by the external ram pressure compression. Murphy et al. (2009) came to the same conclusion investigating the radioFIR relation of perturbed Virgo spiral galaxies based on Spitzer $70 \mu \mathrm{m}$ and VIVA $20 \mathrm{~cm}$ data. They found no local but a slight global radio excess (by a factor of $\sim 2$ ) in NGC 4330, NGC 4388, and NGC 4522. We suggest that this excess is due to a slight compression of the small-scale turbulent magnetic field. On the other hand, the large-scale magnetic field and thus the polarized emission is influenced by the external ram pressure compression. This compression leads to the observed asymmetric ridges of polarized emission. Our result, that the small-scale magnetic field is not significantly compressed whereas the large-scale field is, can be understood in terms of relevant timescales. Whereas the timescale for ram pressure stripping is several $\sim 10 \mathrm{Myr}$ (see, e.g., Vollmer et al. 2001), the free fall time of molecular clouds leading to star formation is several Myr (see, e.g., Krumholz \& Tan 2007). Thus the dynamics of the mainly molecular gas in the star forming spiral arms are decoupled from the overall largescale $(1 \mathrm{kpc})$ motions.

In the same line, the spectral index between 20 and $6 \mathrm{~cm}$ in our sample galaxies only depends on the local star formation rate, as it is the case for unperturbed galaxies (Sukumar et al. 1987; Berkhuijsen et al. 2003; Tabatabaei et al. 2007a,b; Heesen et al. 2009), Sect. 4.3. This is contrary to what we found in NGC 4522 (Vollmer et al. 2004a,b) where the radio continuum spectrum flattens toward the edge of the disk where the gas is compressed. Since NGC 4522 is close to peak ram pressure (Vollmer et al. 2006), a flattening of the spectrum in the compressed region might only occur in galaxies undergoing very strong ram pressure stripping. Unfortunately, the quality of the $20 \mathrm{~cm}$ data of the second galaxy in our sample which is close to peak ram pressure, NGC 4438, does not permit to determine the spectral index within the galactic disk.

We thus conclude that ram pressure by the intracluster medium leads to gas compression on large scales ( $\gtrsim 1 \mathrm{kpc}$; see also Vollmer et al. 2008 for a detailed discussion of NGC 4501) without a significant enhancement of the star formation and the associated small-scale turbulent magnetic field. This is consistent with the results of Koopmann \& Kenney (2004) who found that only $2 \%$ of their 52 Virgo cluster spiral galaxies have an enhanced star formation distribution. Only one galaxy shows a truncated and enhanced star formation distribution. NGC 4321, NGC 4501, and NGC 4522 are classified as having truncated normal star formation distributions, NGC 4535 and NGC 4654 as having normal star formation distributions.

\subsection{The degree of polarization gives additional information on the nature of the interaction}

We suggest that the vertically and azimuthally asymmetric increase of the degree of polarization (Sect. 4.4) is due to ram pressure stripping and thus indicates the ram pressure wind direction 
or the direction of the galaxy's motion in a static intracluster medium. This is consistent with Murphy et al. (2009) who found radio deficits with respect to the FIR surface brightness in the regions where we observe an enhanced degree of polarization in NGC 4402 and NGC 4522 (Vollmer et al. 2004a,b). The absence of a local radio-deficit region at the southern edge of NGC 4388's disk is probably due to the radio continuum emission from the nuclear outflow (Veilleux et al. 1999).

In NGC 4388 (Vollmer \& Huchtmeier 2003), NGC 4402 (Crowl et al. 2005), and NGC 4501 (Vollmer et al. 2008) the degree of polarization is highest in the direction of the ram pressure wind. In unperturbed spiral galaxies galactic rotation leads to an azimuthally symmetric large-scale magnetic field. Due to beam depolarization the degree of polarization decreases towards the edge of the galactic disk in highly inclined galaxies. We interpret the increase of the degree of polarization along the major axis in NGC 4396 and NGC 4402 as ionized ISM that deviates significantly from galactic rotation. In NGC 4402 the western maximum of polarized emission thus traces ram pressure stripped ionized gas with its associated magnetic field. This is consistent with the ram pressure scenario of Crowl et al. (2005) where the ram pressure wind comes from the southeast. By extrapolating this interpretation to NGC 4396, we suggest that the ram pressure wind direction is south.

\section{Conclusions}

Deep VLA 20 and $6 \mathrm{~cm}$ radio continuum data including polarization of a sample of 8 Virgo spiral galaxies are presented and combined with optical DSS, VIVA HI (Chung et al. 2009), and Goldmine $\mathrm{H} \alpha$ data. We study the spatial distributions of the spectral index, the degree of polarization, and the rotation measure and derive the following conclusions:

1. Ram pressure leads to sharp edges of the total power distribution on one side of the galactic disk (NGC 4501, NGC 4654). The radio continuum edge coincides with the HI edge.

2. In edge-on galaxies, the extraplanar radio continuum emission (total power and polarized intensity) can extend further than the HI emission. The most prominent example is NGC 4438.

3. In the case of edge-on galaxies, we find azimuthally asymmetric distributions of the degree of polarization (NGC 4388, NGC 4396, NGC 4402). This asymmetry gives important information on the ram pressure wind direction.

4. Ram pressure does not alter the local total power emission nor the spectral index. Only very strong ram pressure might lead to a flattening of the radio continuum spectrum. This means that the local star formation and the small-scale turbulent magnetic field, which is sensitive to star formation in unperturbed galaxies, are not influenced by external ram pressure compression. We thus conclude that ram pressure by the intracluster medium leads to compression on largescales ( $\geq 1 \mathrm{kpc}$ ). In addition, the absence of enhanced total power emission also implies that star formation is not significantly enhanced in the compressed regions.

Ultimately, we would like to observe these galaxies at $3 \mathrm{~cm}$ in polarized emission to produce reliable rotation measure maps between 3 and $6 \mathrm{~cm}$. Furthermore, single-dish observations at $3 \mathrm{~cm}$ and $6 \mathrm{~cm}$ are needed to fill the missing spacings of interferometric observations. These will help us to discriminate between the 2 creation scenarios of the polarized ridges: in the case of shear or compression of a random field, the resulting ordered field is anisotropic and has no Faraday rotation; whereas in the case of a large-scale magnetic field amplification by compression or shear Faraday rotation is expected to be high.

Acknowledgements. This research has made use of the GOLD Mine Database. This work was supported by the Polish-French (ASTRO-LEA-PF) cooperation program and by the Polish Ministry of Sciences and Higher Education grant $3033 / \mathrm{B} / \mathrm{H} 03 / 2008 / 35$. The authors would like to thank E.M. Berkhuijsen for careful reading of the manuscript.

\section{References}

Beck, R. 2001, Space Sci. Rev., 99, 243

Beck, R. 2005, Cosmic Magnetic Fields, ed. R. Wielebinski, \& R. Beck. (Berlin: Springer), Lect. Notes Phys., 664, 41

Beck, R. 2007, A\&A, 470, 539

Berkhuijsen, E. M., Beck, R., \& Hoernes, P. 2003, A\&A, 398, 937

Byrd, G., \& Valtonen, M. 1990, ApJ, 350, 89

Cayatte, V., van Gorkom, J. H., Balkowski, C., \& Kotanyi, C. 1990, AJ, 100, 604 Chung, A., van Gorkom, J. H., Kenney, J. D. P., Crowl, H. H., \& Vollmer, B. 2009, AJ, 138, 1741

Chyży, K. T. 2008, A\&A, 482, 755

Chyży, K. T., Soida, M., Bomans, D. J., et al. 2006, A\&A, 447, 465

Chyży, K. T., Ehle, M., \& Beck, R. 2007, 474, 415

Crowl, H. H., Kenney, J. D. P., van Gorkom, J. H., \& Vollmer, B. 2005, AJ, 130, 65

Dahlem, M., Lisenfeld, U., \& Rossa, J. 2006, A\&A, 457, 121

Dumke, M., \& Krause, M. 1998, ed. D. Breitschwerdt, M. J. Freyberg, \& J. Truemper, Proc. IAU Colloq., 166, 555

Ehle, M., \& Beck, R. 1993, 273, 45

Gavazzi, G., \& Boselli, A. 1999, A\&A, 343, 93

Gavazzi, G., Boselli, A., \& Kennicutt, R. 1991, AJ, 101, 1207

Gavazzi, G., Boselli, A., Donati, A., Franzetti, P., \& Scodeggio, M. 2003, A\&A, 400,451

Gioia, I. M., Gregorini, L., \& Klein, U. 1982, A\&A, 116, 164

Gunn, J. E., \& Gott, J. R. III 1972, ApJ, 176, 1

Heesen, V., Beck, R., Krause, M., \& Dettmar, R.-J. 2009, A\&A, 494, 563

Hummel, E., \& Saikia, D. J. 1991, A\&A, 249, 43

Hummel, E., van Gorkom, J. H., \& Kotanyi, C. G. 1983, ApJ, 267, L5

Kenney, J. D. P., Rubin, V. C., Planesas, P., \& Young, J. S. 1995, ApJ, 438, 135

Klein, U. 1990, in Windows on Galaxies, ed. G. Fabbiano, J. S. Gallagher, \& A

Renzini (Dordrecht: Kluwer), Ap\&SS Library, 160., 157

Koopmann, R. A., \& Kenney, J. D. P. 2004, ApJ, 613, 866

Krumholz, M. R., \& Tan, J. C. 2007, ApJ, 654, 304

Moore, B., Lake, G., \& Katz, N. 1998, ApJ, 495, 139

Murphy, E. J., Helou, G., Kenney, J. D. P., Armus, L., \& Braun, R. 2008, ApJ, 678,828

Murphy, E. J., Kenney, J. D. P., Helou, G., Chung, A., \& Howell, J. H. 2009, ApJ, 694, 1435

Neininger, N. 1992, A\&A, 263, 30

Neininger, N., Beck, R., Sukumar, S., \& Allen, R. J. 1993, A\&A, 274, 687

Niklas, S., Klein, U., \& Wielebinski, R. 1995, A\&A, 293, 56

Oosterloo, T., Fraternali, F., \& Sancisi, R. 2007, AJ, 134, 1019

Phookun, B., \& Mundy, L. G. 1995, ApJ, 453, 154

Soida, M., Otmianowska-Mazur, K., Chyży, K., \& Vollmer, B. 2006, A\&A, 458, 727

Sukumar, S., \& Allen, R. J. 1991, ApJ, 382, 100

Sukumar, S., Klein, U., \& Gräve, R. 1987, A\&A, 184, 71

Tabatabaei, F. S., Krause, M., \& Beck, R. 2007a, A\&A, 472, 785

Tabatabaei, F. S., Beck, R., Krgel, E., et al. 2007b, A\&A, 475, 133

Tüllmann, R., Dettmar, R.-J., Soida, M., Urbanik, M., \& Rosa, J. 2000, A\&A, 364, L36

Valluri, M. 1993, ApJ, 408, 57

Veilleux, S., Bland-Hawthorn, J., Cecil, G., Tully, R. B., \& Miller, S. T. 1999, ApJ, 520, 111

Völk, H. J., \& Xu, C. 1994, IR Phys. Tech., 35, 527

Vollmer, B., \& Huchtmeier, W. 2003, A\&A, 406, 427

Vollmer, B., Cayatte, V., Balkowski, C., \& Duschl, W. J. 2001, ApJ, 561, 708

Vollmer, B., Thierbach, M., \& Wielebinski, R. 2004a, A\&A, 418, 1

Vollmer, B., Beck, R., Kenney, J. P. D., \& van Gorkom, J. H. 2004b, AJ, 127, 3375

Vollmer, B., Braine, J., Combes, F., \& Sofue, Y. 2005, A\&A, 441, 473

Vollmer, B., Soida, M., Otmianowska-Mazur, K., et al. 2006, A\&A, 453, 883

Vollmer, B., Soida, M., Beck, R., et al. 2007, A\&A, 464, L37

Vollmer, B., Soida, M., Chung, A., et al. 2008, A\&A, 483, 89

Vollmer, B., Soida, M., Chung, A., et al. 2009, A\&A, 496, 669

Weżgowiec, M., Urbanik, M., Vollmer, B., et al. 2007, A\&A, 471, 93 